\title{
Modeling the flow behavior of aluminum alloy 5052
}

\author{
Junhang Guo ${ }^{\mathrm{a}}{ }^{*}$, Yanli Li ${ }^{\mathrm{b}}$ and Hongmin Ding ${ }^{\mathrm{c}}$ \\ Northwest Institute of Mechanical \& Electrical Engineering, No.5, Biyuan East Road, Xianyang, \\ 712099, China \\ aguojunhang@163.com, ${ }^{b}$ marrylea@163.com, ${ }^{c}$ dinghm0630@126.com
}

Keywords: Aluminum alloy 5052, Isothermal hot compression, Constitutive model

Abstract. The deformation behavior of aluminum alloy 5052 is studied by isothermal hot compression in a wide range of temperatures ranging from $300{ }^{\circ} \mathrm{C}$ to $450{ }^{\circ} \mathrm{C}$ and strain rates ranging from $0.01 \mathrm{~s}^{-1}$ to $1 \mathrm{~s}^{-1}$. The results show that the peak stress increases with the strain rate while decreases with the temperature. In the early stage of deformation, the stress increases with the strain to peak stress, while decreases with softening. In this paper, the flow behavior is modeled by a liner function including the peak stress and stress softening rate respect to strain. The effect of strain rate and temperature on the peak flow stress was established by the Arrhenius-type equation. The softening rate was modeled empirically by a multiple linear function of strain rate and temperature. The model parameters were obtained by regressions. The constitutive model was verified and good agreement was achieved.

\section{Introduction}

Aluminum alloys are widely used in modern industry. The study on material's mechanical behavior is essential for application. The constitutive model is the relationship of stress with strain, strain rate and temperature when materials deform subjected to loading. Therefore, a thorough investigation on the material deformation behavior under different temperatures and strain rates is necessary. So, great efforts have been made on the constitutive models in the past decades [1-4].

The typical researches are [5,6] in which the relationship of flow stress, temperature and strain rates was established, though the effect of strain was not considered. Johnson-Cook (JC) model [7] and Zerilli-Armstrong (ZA) model [8] are the widely accepted models describing strain hardening properties. JC model is empirical and ZA model is dislocation-mechanics based. In ref [9], a strain softening function was proposed by the volume fraction of dynamic recrystallization and was employed to model the behavior of some alloys. The modified three parameters Voce type function was proposed in $[10,11]$ for aluminum alloys, in which the effect of deformation condition of the parameters are modeled by multiple linear function. In this paper, the constitutive equation of aluminum alloy 5052 under isothermal hot compression is modeled and verified.

\section{Experiments}

Aluminum alloy 5052 is selected, which is used in aircraft for good plasticity and corrosion resistance. The chemical composition is given in Table 1. Cylindrical specimens with a diameter of 10 $\mathrm{mm}$ and a height of $15 \mathrm{~mm}$ were machined to carry out isothermal hot compression tests by a Gleeble-3500 simulator at temperatures and strain rates .

\begin{tabular}{ccccccccccr}
\multicolumn{1}{c}{ Table } & Chemical composition (in wt.\%) of Al alloy 5052. \\
\hline $\mathrm{S}$ & $\mathrm{F}$ & $\mathrm{C}$ & $\mathrm{M}$ & $\mathrm{M}$ & $\mathrm{C}$ & $\mathrm{Z}$ & $\mathrm{T}$ & $\mathrm{f}$ \\
$\mathrm{i}$ & $\mathrm{e}$ & $\mathrm{u}$ & $\mathrm{n}$ & $\mathrm{g}$ & $\mathrm{r}$ & $\mathrm{n}$ & $\mathrm{i}$ & 1 \\
0 & 0 & 0 & 0 & 2 & 0 & 0 & 0 & $\mathrm{~F}$ \\
.08 & .19 & .02 & .02 & .36 & .18 & .03 & .01 & $\mathrm{e}$ \\
\hline
\end{tabular}

The true strain and stress obtained in tests are:

$$
\varepsilon=\ln \left(\frac{h_{0}}{h}\right)
$$




$$
\sigma_{\text {true }}=\left|\frac{P h}{\pi r_{0}^{2} h_{0}}\right|
$$

Here, $P$ is the load, $h$ is the height after compression, $h_{0}$ and ${ }^{r_{0}}$ are the height and radius after compression. Figure 1 shows the stress strain curves obtained at different temperatures and strain rates. The results show that the flow stress increased with strain rate while decreased with temperature, showing a peak flow stress at certain strain and declines due to recovery/recrystallization.
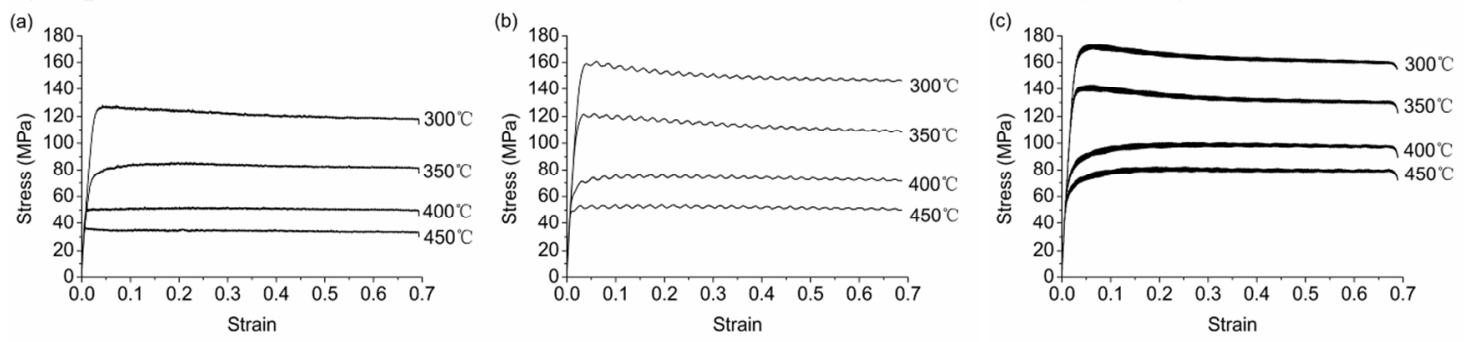

Fig. 1. The measured true stress-true strain curves for $\mathrm{Al}$ alloy 5052: (a) $\& \&=0.01 \mathrm{~s}^{-1}$, (b) $8 \&=0.1 \mathrm{~s}^{-1}$, (c) $8 \&=1 \mathrm{~s}^{-1}$.

Figure 2 present the shape before and after test, complying that Eq. (2) is not accurate for friction $[12,13]$. By ref[14,15], the stress strain curve and be modified by the shape of the specimen:

$$
\sigma_{\text {flow }}=\frac{\sigma_{\text {true }}}{1+\frac{2}{3 \sqrt{3}} m \frac{r_{0}}{h_{0}} \exp \left(\frac{3}{2} \varepsilon\right)}
$$

Here $\sigma_{\text {flow }}$ is the corrected stress, $m$ is the friction factor as a function of dimension,

$$
m=\frac{(R / h) b_{m}}{(4 / \sqrt{3})-\left(2 b_{m} / 3 \sqrt{3}\right)}
$$

$R$ is the equivalent radius assuming volume constant,

$$
R=r_{0} \sqrt{\frac{h_{0}}{h}}
$$

$b_{m}$ is the measuring parameter for deformed specimen,

$$
b_{m}=4 \cdot \frac{R_{M}-R_{T}}{R} \cdot \frac{h}{h_{0}-h}
$$

Here $R_{T}$ is the top radius of deformed specimen which is calculated by the maximum radius $R_{M}$,

$$
R_{T}=\sqrt{3 \frac{h_{0}}{h} r_{0}^{2}-2 R_{M}^{2}}
$$

By corrected as Eq. (3), the stress strain curves are shown in Fig. 3. It can be concluded that the corrected curves are below than measured. The difference between them increase with the strain.

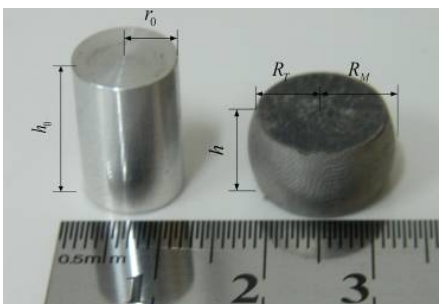

Fig. 2. The configuration of specimen in compression test.
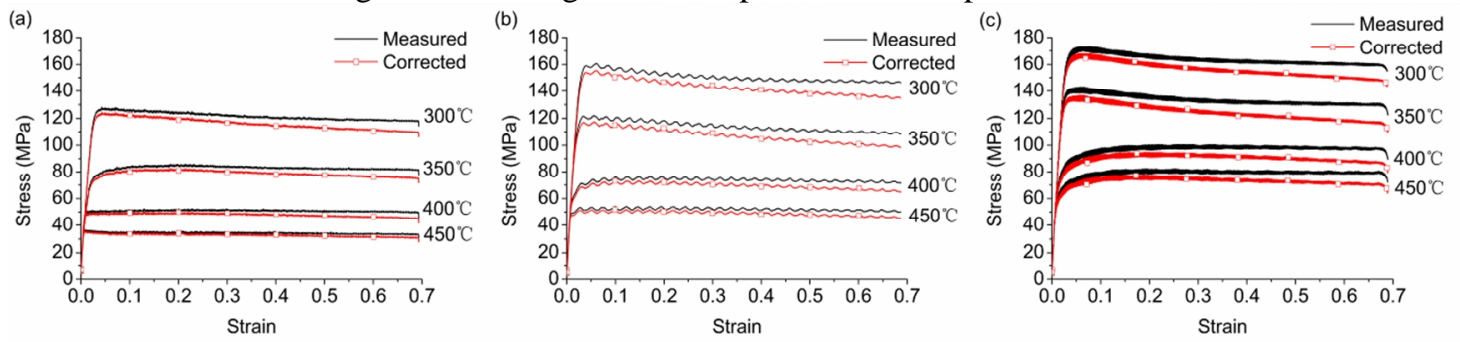

Fig. 3. The corrected and measured flow stress for Al alloy 5052: (a) $8 \&=0.01 \mathrm{~s}^{-1}$, (b) $8 \&=0.1 \mathrm{~s}^{-1}$, (c) $\& \&=1 \mathrm{~s}^{-1}$. 


\section{Constitutive model}

According to the research by Zener and Hollomon[5], the peak flow stress $\sigma$ under hot deformation is influenced by strain rate $8 \delta$ and temperature $T$,

$$
\sigma=\sigma(\& T)
$$

However, in Eq. (9) the effect of strain is neglect, so plenty of constitutive equations with strain compensation were proposed recently [9-11]. According to the characteristics of aluminum alloys, the strain softening behavior due to recovery/recrystallization could be expressed as a linear function of peak flow stress $\sigma$ and strain $\varepsilon$ in this paper:

$$
\sigma_{\text {flow }}=\sigma-k\left(\varepsilon-\varepsilon_{0}\right)
$$

Here $k=\mathrm{d} \sigma_{\text {flow }} / \mathrm{d} \varepsilon$ is the strain softening modulus, $\varepsilon_{0}$ is the strain with respect to peak stress. Because the deformation of metal is effected by activation energy, $Z$ parameter was introduced as a function of strain rate $\&$ and temperature $T[5,6]$ :

$$
Z=\varepsilon \exp \left(\frac{Q}{R T}\right)
$$

Here, $R$ is the universal gas constant $\left(8.3145 \mathrm{Jmol}^{-1} \mathrm{~K}^{-1}\right), Q$ is the activation energy $\left(\mathrm{J} \mathrm{mol}^{-1}\right)$. According to ref [6], for the lower stress level $(\alpha \sigma<0.8)$, the relation between the peak flow stress $\sigma$ and $Z$ parameter is the power law:

$$
Z=A_{1} \sigma^{n}
$$

While for higher stress level $(\alpha \sigma>1.2)$ is the exponential law:

$$
Z=A_{2} \exp (\beta \sigma)
$$

And across the entire range of stress, an Arrhenius-type hyperbolic equation was proposed as:

$$
\begin{aligned}
& Z=A[\sinh (\alpha \sigma)]^{n} \\
& \alpha=\beta / n
\end{aligned}
$$

Here, $A_{1}, A_{2}, A, \alpha, n, \beta$ are the materials constants. By substituting Eq. (13) into Eq. (10) gives

$$
\varepsilon \&=A[\sinh (\alpha \sigma)]^{n} \exp \left(-\frac{Q}{R T}\right)
$$

Then by some algebraic operation, the peak flow stress $\sigma$ can be written as a function of $Z$

$$
\sigma=\left(\frac{1}{\alpha}\right) \ln \left\{\left(\frac{Z}{A}\right)^{1 / n}+\left[\left(\frac{Z}{A}\right)^{2 / n}+1\right]^{1 / 2}\right\}
$$

For some alloys, when the stress reaching peak, the stress declines due to recovery/recrystallization[9-11]. The strain softening rate could be established by a multiple linear function of temperature and strain rate on as Eq. (17) in [10]:

$$
k=k_{0}+k_{1} T+k_{2} \ln 8 \&+k_{3} T \ln 8 \delta
$$

Here $k_{i}, i=0,1,2,3$ are material constants. So the constitutive equations $(9,10,16,17)$ containing a linear strain softening and peak stress was concluded below:

$$
\left\{\begin{array}{c}
\sigma_{\text {flow }}=\sigma-k\left(\varepsilon-\varepsilon_{0}\right) \\
k=k_{0}+k_{1} T+k_{2} \ln \& \&+k_{3} T \ln \& \alpha \\
Z=\varepsilon \exp \left(\frac{Q}{R T}\right) \\
\sigma=\left(\frac{1}{\alpha}\right) \ln \left\{\left(\frac{Z}{A}\right)^{1 / n}+\left[\left(\frac{Z}{A}\right)^{2 / n}+1\right]^{1 / 2}\right\}
\end{array}\right.
$$




\section{Results and discussion}

The corrected flow stress in Fig. 3 was used to fit the peak stress $\sigma$ and softening modulus $k$ by linear regression in Eq. (9) assuming that $\varepsilon_{0}=0.1$. The relation among softening modulus $k$ and temperature $T$, strain rate $8 \delta$ can be plotted in Fig 4 . The effect of temperature and strain rate on softening modulus is monotonous. At a certain temperature, softening modulus increased with strain rate. While for a given stain rate, softening modulus decreased with temperature. The comprehensive effect of temperature and strain rate on softening modulus is bilinear [22,23]. By nonlinear regression, $k_{0}=108.27, k_{1}=-0.1368, k_{2}=5.0627, k_{3}=-0.0053$. The fitting result show that this phenomenon can be modeled by Eq.(17).

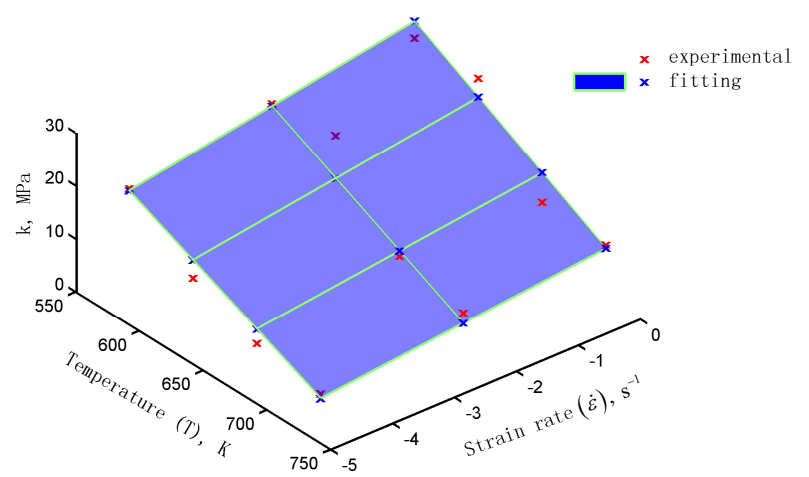

Fig. 4. The influence of $T$ and $\& \delta$ upon the softening coefficient $k$ and the multiple linear regression result.

In order to obtain the correlation of the peak flow stress $\sigma$ with temperature $T$ and strain rate $8 \delta$, substituting Eq. (10) into Eq.(11) and Eq.(12), the power law equation and the exponential law equation can be get as:

$$
\begin{aligned}
& \delta \&=A_{1} \sigma^{n} \exp \left(-\frac{Q}{R T}\right) \\
& \& \& A_{2} \exp (\beta \sigma) \exp \left(-\frac{Q}{R T}\right)
\end{aligned}
$$

By taking logarithms of both sides, Eq. (19) and Eq. (20) can be rewritten as:

$$
\begin{aligned}
& \ln \& \ln A_{1}+n \ln \sigma-Q /(R T) \\
& \ln \& \ln A_{1}+\beta \sigma-Q /(R T)
\end{aligned}
$$

It can be seen that $n$ and $\beta$ under different temperature can be obtained from the slope of the lines in the $\ln \& \& \ln \sigma$ and $\ln \& \alpha \sigma$ plot, respectively. By linear regression of Fig.5, a series of $n, \beta$ and $\alpha$ can be get. The $\alpha \sigma$ values can be used to distinguish the stress level.
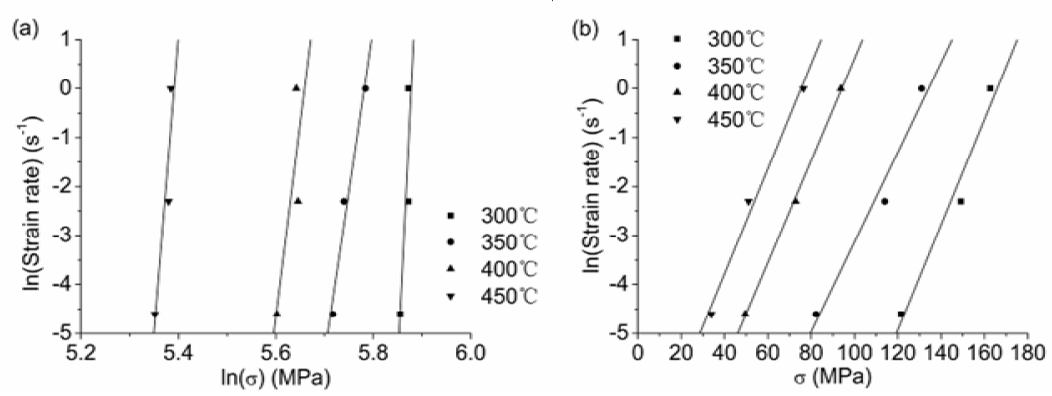

Fig. 5. Relationships between strain rate and peak flow stress: (a) $\ln 8 \delta \ln \sigma$, (b) $\ln 8 \delta \sigma$.

According to Eq. (21) and Eq.(22), for a given stain rate $\delta, n R / Q$ and $\beta R / Q$ can be represented as the slope of $\ln \sigma-1 / T$ plot and $\sigma-1 / T$ plot. By linear fitting as shown in Fig. 6, the value of $\alpha$ can determined

$$
\alpha=\beta / n=0.01615
$$

Taking the logarithm of both sides of the Eq. (20) gives

$$
\ln \& \ln A-Q /(R T)+n \ln [\sinh (\alpha \sigma)]
$$


Differentiating Eq. (24) gives that

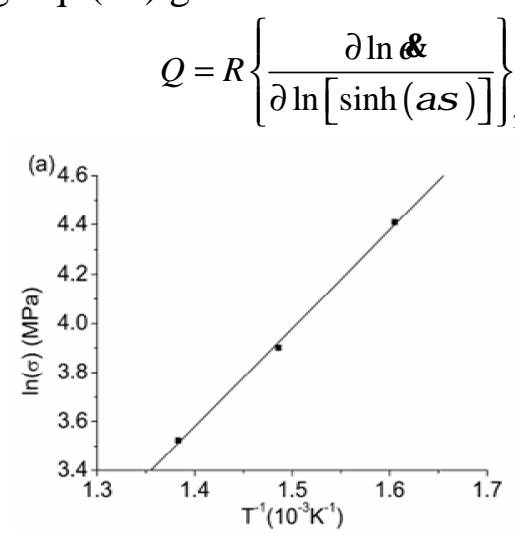
$\left\{\frac{\partial \ln [\sinh (\alpha \sigma)]}{\partial(1 / T)}\right\}$

Fig. 6. Relationships between peak flow stress and temperature: (a) $\ln \sigma-1 / T$, (b) $\sigma-1 / T$.

By substitute the $\alpha$ value, the relation of $\ln \varepsilon \alpha \ln [\sinh (\alpha \sigma)]$ and $\ln [\sinh (\alpha \sigma)]-1 / T$ can be obtained as shown in Fig. 7 and Fig. 8 respectively. The value of $Q$ can be obtained from the multiply of the slopes of $\ln \& \alpha \ln [\sinh (\alpha \sigma)]$ and $\ln [\sinh (\alpha \sigma)]-1 / T$ plots and calibrated as $Q=213.141 \mathrm{~kJ} \cdot \mathrm{mol}^{-1}$.
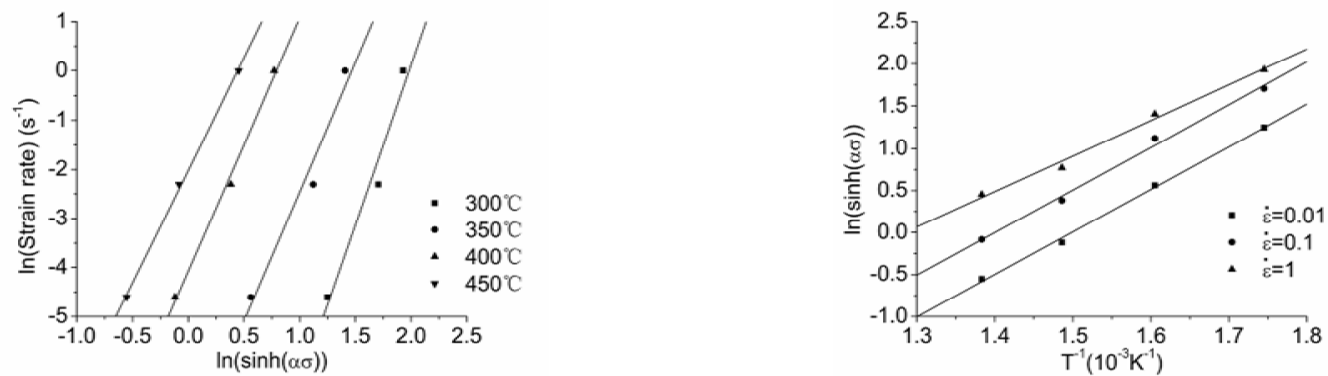

Fig. 7. Relationships between $\ln \& \& \ln [\sinh (\alpha \sigma)]$. Fig. 8. Relationships between $\ln [\sinh (\alpha \sigma)]-1 / T$.

Taking the logarithm of both sides of the Eq. (13) gives

$$
\ln Z=\ln A+n \ln [\sinh (\alpha \sigma)]
$$

The relation between $\ln Z$ and $\ln [\sinh (\alpha \sigma)]$ is linear and can be shown in Fig. 8. The values of $\ln A$ and $n$ can be obtained by the intercept and slope of the $\ln Z-\ln [\sinh (\alpha \sigma)]$ plots. Then the value of $A$ and $n$ can be calibrated as $A=4.1903 \times 10^{14} \mathrm{~s}^{-1}$ and $n=5.313$ respectively. Then all the material parameters are concluded in Table 2.

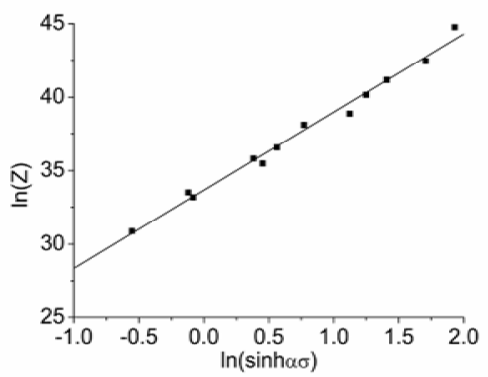

Fig. 9. Relationships between $\ln Z-\ln [\sinh (\alpha \sigma)]$.

The validity of the novel constitutive equations for aluminum alloy 5052, comparisons between the experimental and predicted flow stress were carried out as shown in Fig. 10. It can be seen that good agreement with the experiments was achieved. In Fig.11, the coefficient is 0.9983, implying good agreement. 

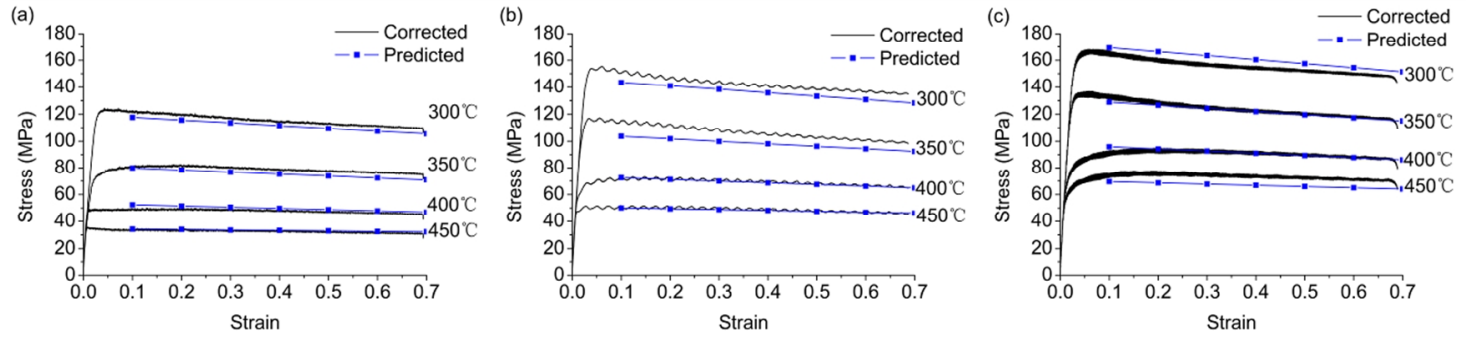

Fig. 10. Comparisons between predicted and experimental: (a) $\& \&=0.01 \mathrm{~s}^{-1}$,(b) $\& \&=0.1 \mathrm{~s}^{-1}$, (c) $8 \&=1 \mathrm{~s}^{-1}$.

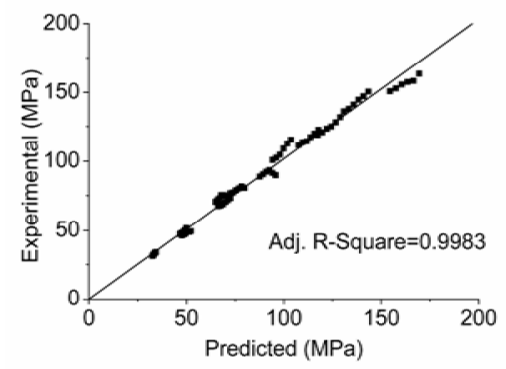

Fig. 11. Correlation between the experimental (corrected) and predicted flow stresses obtained from the new model.

Table 2 Parameters and values of novel constitutive equation

\begin{tabular}{cccc}
\hline$A / \mathrm{s}^{-1}$ & $n$ & $Q / \mathrm{kJ} \times \mathrm{mol}^{-1}$ & $\alpha$ \\
\hline $4.1903 \times 10^{14}$ & 5.313 & 213.141 & $\begin{array}{c}\mathbf{0 . 0 1 6 1} \\
\mathbf{5}\end{array}$ \\
\hline$k_{0}$ & $k_{1}$ & $k_{2}$ & $k_{3}$ \\
\hline 108.27 & $\begin{array}{c}-0.136 \\
8\end{array}$ & 5.0627 & $\mathbf{- 0 . 0 0 5 3}$ \\
\hline
\end{tabular}

\section{Conclusions}

The isothermal hot compression tests of aluminum alloy 5052, in a wide range of temperatures ranging from $300{ }^{\circ} \mathrm{C}$ to $450{ }^{\circ} \mathrm{C}$ and strain rates ranging from $0.01 \mathrm{~s}^{-1}$ to $1 \mathrm{~s}^{-1}$ were employed to study its high-temperature flow behavior.

1) A novel constitutive equation combining a linear softening term and peak stress was proposed to describe the material behavior.

2) The peak flow stress were represented by Zener-Holloman parameter including Arrhenius term.

3) The effects of strain rate and temperature on the softening modulus was analyzed empirically and regressed by a linear function.

4) The validity of the novel constitutive equations was verified by comparing the experimental results and predicted results, and good agreement was achieved.

\section{References}

[1] Riqiang Liang, Akhtar S. Khan, Int. J. Plast 15 (1999) 963-980.

[2] Z. Gronostajski, J. Mater. Proc. Technol. 106 (2000) 40-44.

[3] H.J. McQueen, N.D. Ryan, Mater. Sci. Eng. A 322 (2002) 43-63.

[4] Taku Sakai, Andrey Belyakov, Rustam Kaibyshev, Hiromi Miura, John J. Jonas, Prog. Mater. Sci. 60 (2014) 130-207.

[5] C. Zener, J.H. Hollomon, J. Appl. Phys. 15 (1944) 22.

[6] C.M. Sellars, W.J. McTegart, Acta Metall. 14 (1966) 1136.

[7] G. R. Johnson, W. H. Cook, Eng. Fract. Mech., 21(1985) 31-48.

[8] F. J. Zerilli, R. W. Armstrong: J. Appl. Phys., 1987, 61, 1816.

[9] C.M. Sellars, Czech. J. Phys. B 35 (1985) 239-248.

[10] J.H. Guo, S.D. Zhao, G.H. Yan, Z.B. Wang, Mater. Sci. Technol. 29 (2013) 197-203.

[11] J.H. Guo, S.D. Zhao, R. Murakami, R.X. Ding, S.Q. Fan, J. Alloys Comp. 566 (2013) 62-67. 
[12] R.W. Evans, P.J. Scharning, Mater. Sci. Technol. 17 (2001) 995-1004.

[13] R. Ebrahimi, A. Najafizadeh, J. Mater. Proc. Technol. 152 (2004) 136-143.

[14] A. Gholamzadeh, A. Karimi Taheri, Mech. Res. Commun. 36 (2009) 252-259.

[15] W.T. Read, Dislocation in Crystal, McGraw Hill, New York, 1953. 